BNL Informal Report

BNL - 75019-2005-IR

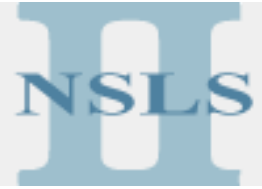

Simulation of Transverse Instabilities in the NSLS II Storage Ring

S. Krinsky

National Synchrotron Light Source

Brookhaven National Laboratory

Upton, New York 11973

September, 2005 


\section{DISCLAIMER}

This report was prepared as an account of work sponsored by an agency of the United States Government. Neither the United States Government nor any agency thereof, nor any of their employees, nor any of their contractors, subcontractors or their employees, makes any warranty, express or implied, or assumes any legal liability or responsibility for the accuracy, completeness, or any third party's use or the results of such use of any information, apparatus, product, or process disclosed, or represents that its use would not infringe privately owned rights. Reference herein to any specific commercial product, process, or service by trade name, trademark, manufacturer, or otherwise, does not necessary constitute or imply its endorsement, recommendation, or favoring by the United States Government or any agency thereof or its contractors or subcontractors. The views and opinions of authors expresses herein do not necessarily state to reflect those of the United States Government or any agency thereof. 


\title{
Simulation of Transverse Instabilities in the NSLS-II Storage Ring*
}

\author{
S. Krinsky \\ NSLS, Brookhaven National Laboratory, Upton, NY 11973
}

\begin{abstract}
We have carried out computer simulations using MATLAB to study the transverse coupled-mode instability (TMCI) in the proposed NSLS-II storage ring. Our calculations have been performed using transverse short-range wakefields describing: a broad-band resonator; a resistive wall with normal surface impedance; and a chamber wall with extreme anomalous skin effect. We have considered: (1) the ring with a single-frequency RF system for which the equilibrium longitudinal bunch distribution is Gaussian; and (2) the ring with a third harmonic (Landau) cavity included to lengthen the bunch. Based on current NSLS-II design parameters, we report estimates of the TMCI threshold behavior.
\end{abstract} All of our results for the instability threshold (at zero chromaticity) are consistent (to about $\pm 30 \%$ ) with the simple relation

$$
\frac{e^{2} N_{e}^{t h} \beta_{y}}{4 \pi \gamma m c^{2} v_{s}} \kappa_{\perp} \cong 0.7,
$$

where $\kappa_{\perp}$ is the transverse kick factor, $N_{e}^{\text {th }}$ is the number of electrons in the bunch at threshold, $\beta_{y}$ is the vertical betafunction at the impedance, and $\gamma m c^{2}$ is the electron energy. For a single-frequency RF system, $v_{s}$ is the synchrotron tune, while for a Landau cavity, $v_{s}$ is the synchrotron tune of an electron with synchrotron amplitude equal to the rms bunch length.

* This work was supported by Department of Energy contract DE-AC02-98CH10886. 
Contents

1. Introduction

2. Equations of Motion

3. Threshold Condition for Transverse Coupled Mode Instability

4. Parameters for NSLS-II

5. Simulation Results: Broad-Band Resonator, Fundamental RF

6. Simulation Results: Resistive Wall, Fundamental RF

7. Simulation Results: Extreme Anomalous Skin Effect

8. Landau Cavity

9. Simulation Results: Broad-Band Resonator, Landau Cavity

10. Simulation Results: Resistive Wall, Landau Cavity

11. Simulation Results: Extreme Anomalous Skin Effect, Landau Cavity

12. Summary of Results

13. Concluding Remarks

References 


\section{Introduction}

In this note, we describe computer simulations using MATLAB to study transverse instabilities in the proposed NSLS-II storage ring. In order to investigate the single-bunch transverse coupled mode instability (TMCI) [1-8], we have incorporated transverse short-range wakefields [9,10] describing: (1) a broad-band resonator; (2) a resistive wall with normal surface impedance; and (3) a resistive wall with extreme anomalous skin effect. To facilitate study of coupled bunch instabilities, we have included normal and extreme-anomalous long-range resistive wall wakefields coupling different bunches via their transverse center of mass motion. Of special concern in this study are the wakefields arising from warm and superconducting small-gap undulators which are the primary sources of radiation for NSLS-II.

The program provides a description of instabilities in the cases: (1) when the ring has a single-frequency RF system and the equilibrium longitudinal bunch distribution is Gaussian; (2) when there is a higher harmonic (Landau) cavity [11] included to lengthen the bunch. Based on current NSLS-II design parameters, we report estimates of the TMCI threshold behavior. In the case of a broad-band resonator, we have checked that our results are in reasonable agreement with the frequency-domain code MOSES [4].

\section{Equations of Motion}

We consider the motion of $n_{b}$ bunches, each comprised of $n_{p}$ macroparticles, over $n_{r}$ revolutions around the ring. The integrated effect of the wakefield is applied to the beam once per revolution. We denote the revolution period $T_{0}$, angular revolution frequency $\omega_{0}=2 \pi / T_{0}$, momentum compaction $\alpha$, synchrotron oscillation tune $v_{s}=\omega_{s} / \omega_{0}$, vertical betatron tune of the synchronous particle $v_{0 y}$, vertical chromaticity $\xi_{y}$, and the vertical beta-function at the point of wakefield interaction $\beta_{y}$. We introduce the revolution number $i=1, \ldots, n_{r}$, the bunch number, $j=1, \ldots, n_{b}$, and the macroparticle number $k=1, \ldots, n_{p}$. For the $k^{\text {th }}$ macroparticle in the $j^{\text {th }}$ bunch on the $i^{\text {th }}$ revolution: the temporal deviation from the synchronous particle is $\tau_{i}(j, k)$ (positive indicates the macroparticle is in front of the synchronous particle); the fractional energy deviation is 
$\varepsilon_{i}(j, k)$; the vertical displacement is $y_{i}(j, k)$; the vertical angle is $p_{i}(j, k)=y_{i}{ }^{\prime}(j, k)$; and the vertical betatron tune is $v_{y, i}(j, k)$.

The transverse wakefield is denoted $w_{\perp}(s)$. Electron " 1 ” with vertical displacement $y_{1}$ induces a change in vertical angle $\Delta y_{2}$ ' of electron "2" a distance $s$ behind the first, while traveling a distance $\Delta \ell$ on the ring orbit, where

$$
\Delta y_{2}{ }^{\prime}=\frac{e^{2} w_{\perp}(z) \Delta \ell}{\gamma m c^{2}} y_{1} .
$$

We shall write the transverse wake in the form

$$
w_{\perp}(s) \Delta \ell=W_{0} f(s),
$$

where $f(s)$ is a dimensionless function. We define the dimensionless strength parameter,

$$
\mathrm{Y}=\frac{e^{2} N_{e} \beta_{y} W_{0}}{8 \gamma m c^{2} v_{s}},
$$

with $N_{e}$ being the number of electrons in a bunch. The number of electrons per macroparticle is $N_{e} / n_{p}$.

In the case of a single frequency RF system, the equations of motion are given by:

$$
\begin{gathered}
\varepsilon_{i+1}(j, k)=\varepsilon_{i}(j, k)+\frac{\left(2 \pi v_{s}\right)^{2}}{\alpha T_{0}} \tau_{i}(j, k) \\
\tau_{i+1}(j, k)=\tau_{i}(j, k)-\alpha T_{0} \varepsilon_{i+1}(j, k) \\
v_{y, i}(j, k)=v_{0 y}+\xi \varepsilon_{i}(j, k) \\
y_{i+1}(j, k)=y_{i}(j, k) \cos \left[2 \pi v_{y, i}(j, k)\right]+p_{i}(j, k) \beta_{y} \sin \left[2 \pi v_{y, i}(j, k)\right] \\
p_{i+1}(j, k)=-y_{i}(j, k) \frac{1}{\beta_{y}} \sin \left[2 \pi v_{y, i}(j, k)\right]+p_{i}(j, k) \cos \left[2 \pi v_{y, i}(j, k)\right] \\
+\frac{8 v_{s} \mathrm{Y}}{n_{p} \beta_{y}} \sum_{k^{\prime}=1}^{n_{p}} y_{i}\left(j, k^{\prime}\right) f\left[c \tau_{i}\left(j, k^{\prime}\right)-c \tau_{i}(j, k)\right] \\
+\frac{8 v_{s} \mathrm{Y}}{n_{p} \beta_{y}} \sum_{m=1}^{i j-1} y_{i}(i j-m) f\left[\frac{m c T_{0}}{n_{b}}\right] .
\end{gathered}
$$

Here, we have defined

$$
i j=n_{b}(i-1)+j
$$


and

$$
\bar{y}(i j)=\frac{1}{n_{p}} \sum_{k=1}^{n_{p}} y_{i}(j, k) .
$$

Initial values are determined from a Gaussian random number generator: temporal displacements $\tau_{0}(j, k)$ have standard deviation $\sigma_{t}$; energy deviations $\varepsilon_{0}(j, k)$ have standard deviation $\sigma_{\varepsilon}=v_{s} \omega_{0} \sigma_{t} / \alpha$; vertical displacements $y_{0}(j, k)$ have standard deviation $\sigma_{y}=\sqrt{\beta_{y} \varepsilon_{y}}$; and the vertical angular deviations $p_{0}(j, k)$ have standard deviation $\sigma_{y}{ }^{\prime}=\sqrt{\varepsilon_{y} / \beta_{y}}$.

While tracking the particles, for each bunch we save the values of the center of mass displacement, $\bar{y}(i j)$, and the rms deviation $y_{r m s}(i j)$ of the electrons from the center of mass, where

$$
y_{r m s}(i j)=\sqrt{\frac{1}{n_{p}} \sum_{k=1}^{n_{p}}\left[y_{i}(j, k)-\bar{y}(i j)\right]^{2}} .
$$

We carry out a fast Fourier transform of the center of mass displacements and plot the resulting spectral amplitudes in the region near the betatron sidebands.

For a resonator [10], with transverse shunt impedance $R_{\perp}(\mathrm{ohm} / \mathrm{m})$, quality factor $Q_{\perp}$ and resonant frequency $\omega_{r}=k_{r} c$,

$$
f^{r e s}(s)=\exp \left[-\frac{k_{r} s}{2 Q_{\perp}}\right] \sin \left[k_{r} s \sqrt{1-\frac{1}{4 Q_{\perp}^{2}}}\right] \text {, }
$$

and

$$
W_{0}^{r e s}=\frac{\omega_{r} R_{\perp}}{\sqrt{Q_{\perp}^{2}-\frac{1}{4}}}, \quad \mathrm{Y}^{r e s}=\frac{e^{2} N_{e} \beta_{y} W_{0}^{\text {res }}}{8 \gamma m c^{2} v_{s}} .
$$




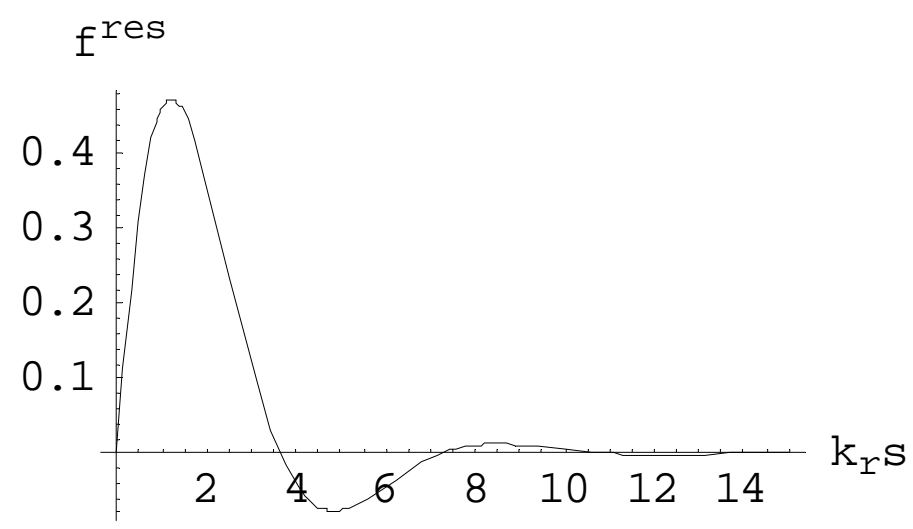

Figure 1. Shape of wakefield for broadband resonator with $Q_{\perp}=1$.

For the normal transverse resistive wall wakefield [12] of a warm circular tube of length $L$, radius $b$ and conductivity $\sigma$,

$$
f^{r \omega}(s)=\frac{4}{3} e^{-z / s_{0}}\left(-\cos \left(\frac{s \sqrt{3}}{s_{0}}\right)+\sqrt{3} \sin \left(\frac{s \sqrt{3}}{s_{0}}\right)\right)+\frac{16 \sqrt{2}}{\pi} \int_{0}^{\infty} \frac{d x e^{-x^{2} s / s_{0}}}{x^{6}+8},
$$

where

$$
s_{0}=\left(\frac{2 b^{2}}{Z_{0} \sigma}\right)^{1 / 3}
$$

and

$$
W_{0}^{r w}=\frac{c Z_{0}}{4 \pi} \frac{2 s_{0} L}{b^{4}}, \quad \mathrm{Y}^{r w}=\frac{e^{2} N_{e} \beta_{y} W_{0}^{r w}}{8 \gamma m c^{2} v_{s}} .
$$

To speed calculations, we use the approximation

$$
f^{r w}(s) \cong \frac{4}{3} e^{-z / s_{0}}\left(-\cos \left(\frac{s \sqrt{3}}{s_{0}}\right)+\sqrt{3} \sin \left(\frac{s \sqrt{3}}{s_{0}}\right)\right)+\frac{1}{\sqrt{2 \pi}} \frac{1}{\left[a^{p}+\left(s / 4 s_{0}\right)^{p / 2}\right]^{1 / p}}
$$

with

$$
a=\frac{3}{4 \sqrt{2 \pi}}, \quad p=2.7
$$

For $s>s_{0}$, 


$$
f^{r w}(s) \approx \sqrt{\frac{2 s_{0}}{\pi s}} .
$$

For a superconducting circular tube the wakefield [13] is determined by the extreme anomalous skin effect and

$$
\begin{aligned}
& f^{\text {ease }}(s)=e^{-z / s_{0}}\left(\frac{3(-3+\sqrt{5})}{5} \cos \left(\sqrt{5+2 \sqrt{5}} s / s_{a}\right)+\frac{3(-1+\sqrt{5}) \sqrt{2(5+\sqrt{5})}}{10} \sin \left(\sqrt{5+2 \sqrt{5}} s / s_{a}\right)\right) \\
& +\frac{3 \sqrt{3}(-1+\sqrt{5})}{10 \pi} \int_{0}^{\infty} d x \frac{\exp \left[-4\left(s / s_{a}\right) x^{3 / 5} /(-1+\sqrt{5})\right]}{x^{3 / 5}\left(1+x+x^{2}\right)}
\end{aligned}
$$

where we define

$$
S_{a}=\frac{(2 \sqrt{3} / \pi)^{1 / 5}}{2 \sin (\pi / 10)}\left(\frac{b^{3}}{\sigma \text { lratio } Z_{0}}\right)^{1 / 5},
$$

and

$$
W_{0}^{\text {ease }}=\frac{c Z_{0}}{4 \pi} \frac{2 s_{a} L}{b^{4}}, \quad \mathrm{Y}^{\text {ease }}=\frac{e^{2} N_{e} \beta_{y} W_{0}^{\text {ease }}}{8 \gamma m c^{2} v_{s}} .
$$

To increase the speed of calculation, we use the approximation

$$
\begin{aligned}
& f^{\text {ease }}(s) \cong e^{-z / s_{0}}\left(\frac{3(-3+\sqrt{5})}{5} \cos \left(\sqrt{5+2 \sqrt{5}} s / s_{a}\right)+\frac{3(-1+\sqrt{5}) \sqrt{2(5+\sqrt{5})}}{10} \sin \left(\sqrt{5+2 \sqrt{5}} s / s_{a}\right)\right) \\
& +\frac{1}{\Gamma(1 / 3)} \frac{1}{\sqrt{a^{2}+\left(d s / s_{a}\right)^{4 / 3}}}
\end{aligned}
$$

with

$$
a=\frac{10}{-6(-3+\sqrt{5}) \Gamma(1 / 3)} \quad d=\frac{4}{(-1+\sqrt{5})^{5 / 2}}
$$

For $z>>S_{a}$,

$$
f^{\text {ease }}(s) \approx \frac{(-1+\sqrt{5})^{5 / 3}}{\Gamma(1 / 3)}\left(\frac{s_{a}}{4 s}\right)^{2 / 3}=\eta\left(\frac{s_{a}}{s}\right)^{2 / 3} .
$$

where

$$
\eta=\frac{4}{\Gamma(1 / 3)}(\sin (\pi / 10))^{5 / 3}=0.21
$$




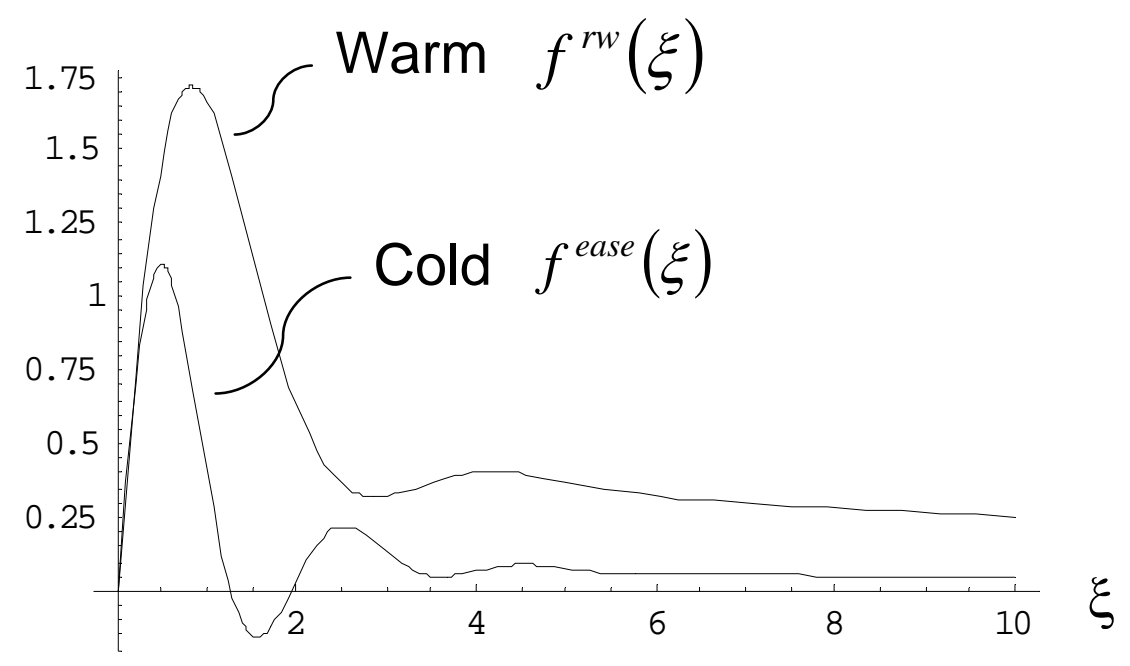

Figure 2. Comparison of shape functions for normal resistive wall wakefield (warm, $\xi=s / s_{0}$ ) and wakefield for extreme anomalous skin effect (cold, $\xi=s / s_{a}$ ). 


\section{Threshold Condition for Transverse Coupled Mode Instability}

The simulations reported in the following sections, support the use of a simple approximation to estimate the threshold of the transverse mode coupling instability. If the center of mass of an electron bunch containing $N_{e}$ electrons has transverse displacement $y_{c m}$ from the axis, the transverse angular kick experienced by its center of mass is given by

$$
y_{c m}{ }^{\prime}=\frac{e^{2} N_{e}}{\gamma m c^{2}} y_{c m} \kappa_{\perp},
$$

where the transverse kick factor $\kappa_{\perp}$ is expressed in terms of the wake field by

$$
\kappa_{\perp}=\int_{-\infty}^{\infty} d s \int_{-\infty}^{s} d s^{\prime} F(s) F\left(s^{\prime}\right) w_{\perp}\left(s-s^{\prime}\right) \Delta \ell,
$$

and $F(s)$ is the normalized bunch density. The kick factor can also be determined in terms of the transverse impedance via

$$
\kappa_{\perp}=\frac{c}{\pi} \int_{0}^{\infty} d k|\tilde{F}(k)|^{2} \operatorname{Im} Z_{\perp}(k),
$$

where $\tilde{F}(k)$ is the Fourier transform of the bunch density normalized by $\tilde{F}(0)=1$.

Let us define an average transverse coherent tune shift (divided by the synchrotron tune $v_{s}$ ) via

$$
\left(\frac{\Delta v_{y}}{v_{s}}\right)^{a v} \equiv \frac{e^{2} N_{e} \beta_{y}}{4 \pi \gamma m c^{2} v_{s}} \kappa_{\perp}=\frac{2}{\pi} \frac{\mathrm{Y}}{W_{0}} \kappa_{\perp} .
$$

For the fundamental RF system, our simulations show that the threshold occurs when $\left(\Delta v_{y} / v_{s}\right)^{a v}$ has a value between 0.45 and 1 . Therefore to an accuracy of about $\pm 30 \%$, the threshold is determined by

$$
\frac{e^{2} N_{e} \beta_{y}}{4 \pi \gamma m c^{2} v_{s}} \kappa_{\perp}=0.7
$$

We have confirmed this by simulations using wakefields corresponding to a broad-band resonator, resistive wall and extreme anomalous skin effect. It is reasonable to expect that this approximation will be useful for more general wakefields. 
The kick factor corresponding to a resonant wakefield can be approximated in the two limits $k_{r} \sigma_{s}<<1$ and $k_{r} \sigma_{s}>>1$. From Eq. (3.2), one finds

$$
\kappa_{\perp}^{r e s} \cong \int_{-\infty}^{\infty} d s \int_{-\infty}^{s} d s^{\prime} F(s) F\left(s^{\prime}\right)\left(s-s^{\prime}\right) w_{\perp}(0+) \Delta \ell, \quad\left(k_{r} \sigma_{s}<<1\right)
$$

and from Eq. (3.3),

$$
\kappa_{\perp}^{r e s} \cong \frac{c}{\pi} \int_{0}^{\infty} d k|\tilde{F}(k)|^{2} \operatorname{Im} Z_{\perp}(0)=c \operatorname{Im} Z_{\perp}(0) \int_{-\infty}^{\infty} d s F^{2}(s) \quad\left(k_{r} \sigma_{s}>>1\right)
$$

For a ring with only the fundamental RF, the longitudinal bunch

$$
\begin{gathered}
\text { distribution } F(s)=\frac{1}{\sqrt{2 \pi} \sigma_{s}} \exp \left(-\frac{s^{2}}{2 \sigma_{s}^{2}}\right) \text {. It then follows from Eqs. (3.6) that } \\
\qquad \kappa_{\perp}^{r e s} \cong \frac{1}{\sqrt{\pi}} \frac{c R_{\perp}}{Q_{\perp}}\left(k_{r}^{2} \sigma_{s}\right) \quad\left(k_{r} \sigma_{s}<<1\right)
\end{gathered}
$$

and frm (3.7) that

$$
\kappa_{\perp}^{r e s} \cong \frac{c}{2 \sqrt{\pi} \sigma_{s}} \frac{R_{\perp}}{Q_{\perp}} \quad\left(k_{r} \sigma_{s}>>1\right)
$$

We see that when $k_{r} \sigma_{s}<<1$, the kick factor increases linearly with bunch length and quadratically with frequency. When $k_{r} \sigma_{s}>1$, the kick factor is inversely proportional to bunch length and independent of frequency.

For the resistive wall wakefield, we use Eqs. (2.18) and (3.2) to approximate the the kick factor by

$$
\kappa_{\perp}^{r w} \cong 0.58 \frac{c Z_{0}}{4 \pi} \frac{2 s_{0} L}{b^{4}} \sqrt{\frac{s_{0}}{\sigma_{s}}}
$$

and for the extreme anomalous skin effect, we use Eqs. (2.24) and (3.2) to show that

$$
\kappa_{\perp}^{\text {ease }} \cong 0.21 \frac{c Z_{0}}{4 \pi} \frac{2 s_{a} L}{b^{4}}\left(\frac{s_{a}}{\sigma_{s}}\right)^{2 / 3}
$$

When a Landau cavity is used, the synchrotron tune depends linearly on the oscillation amplitude. We define $\bar{v}_{s}$ to be the synchrotron frequency of an electron with oscillation amplitude equal to the rms bunch length. From simulation, we find that a useful approximation to the threshold condition is 


$$
\frac{e^{2} N_{e} \beta_{y}}{4 \pi \gamma m c^{2} \bar{v}_{s}} \kappa_{\perp}=0.7,
$$

where the kick factor is evaluated using the appropriate electron distribution:

$F(s)=\hat{N} \exp \left(-\lambda s^{4}\right)$, where $\hat{N}=\frac{2 \lambda^{1 / 4}}{\Gamma(1 / 4)}$, and the rms bunch length $\Sigma_{s}^{2}=\frac{\Gamma(3 / 4)}{\lambda^{1 / 2} \Gamma(1 / 4)}$.

The Landau cavity is operated under conditions such that $\bar{v}_{s}<<v_{s}$ and $\Sigma_{s} \gg>\sigma_{s}$. It follows from Eqs. (3.6) and (3.7) that

$$
\kappa_{\perp}^{r e s} \cong 0.57 \frac{c R_{\perp}}{Q_{\perp}}\left(k_{r}^{2} \Sigma_{s}\right) \quad\left(k_{r} \Sigma_{s}<<1\right)
$$

and

$$
\kappa_{\perp}^{r e s} \cong 0.27 \frac{C}{\Sigma_{s}} \frac{R_{\perp}}{Q_{\perp}} \quad\left(k_{r} \Sigma_{s}>>1\right)
$$

Hence, for a resonant wake field with $k_{r} \Sigma_{s}<<1$, we see from Eqs. (3.4), (3.8), (3.12) and (3.13) that the ratio of the threshold currents with and without the Landau cavity is

$$
\frac{I_{\text {th }}^{\text {Landau }}}{I_{\text {th }}^{\text {fund }}} \cong \frac{\bar{v}_{s}}{v_{s}} \frac{\sigma_{s}}{\Sigma_{s}} .
$$

In this case the Landau cavity can greatly reduce the instability threshold. For a resonant wake with $k_{r} \sigma_{s} \gg>1$, it follows from Eqs. (3.4), (3.9), (3.12) and (3.14) that

$$
\frac{I_{\text {th }}^{\text {Landau }}}{I_{\text {th }}^{\text {fund }}} \cong \frac{\bar{v}_{s}}{v_{s}} \frac{\Sigma_{s}}{\sigma_{s}} .
$$

In this case the Landau cavity has only a small effect on the threshold current since the two factors in Eq. (3.13) approximately offset one another.

For a resistive wall wake, the kick factor with a Landau cavity is

$$
\kappa_{\perp}^{r w} \cong 0.56 \frac{c Z_{0}}{4 \pi} \frac{2 s_{0} L}{b^{4}} \sqrt{\frac{s_{0}}{\Sigma_{s}}},
$$

and for the anomalous skin effect wake

$$
\kappa_{\perp}^{\text {ease }} \cong 0.21 \frac{c Z_{0}}{4 \pi} \frac{2 s_{a} L}{b^{4}}\left(\frac{s_{a}}{\Sigma_{s}}\right)^{2 / 3} .
$$


It then follows that the ratio of the threshold currents with and without the Landau cavity is given by

$$
\frac{I_{\text {th }}^{\text {Landau }}}{I_{\text {th }}^{\text {fund }}} \cong \frac{\overline{v_{s}}}{v_{s}}\left(\frac{\Sigma_{s}}{\sigma_{s}}\right)^{p}
$$

where

$$
p=\left\{\begin{array}{ll}
1 / 2 & \text { for resistive wall } \\
2 / 3 & \text { for extreme anomalous skin effect }
\end{array}\right\} .
$$




\section{Illustrative Parameters for NSLS-II}

Ring

$E=3 G e V$

$T_{0}=2 \mu \mathrm{s}$

$\alpha=1.7 \times 10^{-4}$

$N_{e}=9.4 \times 10^{9}$

$I_{e}=e N_{e} / T_{0}=0.75 \mathrm{ma}$

$\sigma_{\varepsilon}=10^{-3}$

Fundamental $r f$

$f_{r f}=500 \mathrm{MHz} \quad(h=1000)$

$v_{s}=.004$

$\sigma_{\tau}=12 \mathrm{ps}, \quad \sigma_{\mathrm{s}}=3.6 \mathrm{~mm}$

Insertion Device

$\beta_{y}=3 m$

$L=20 \times 5 m$

$b=2.5 \mathrm{~mm}$

warm chamber

$\sigma=6 \times 10^{7}(\mathrm{ohm}-\mathrm{m})^{-1}$

$s_{0}=8 \mu \mathrm{m}$

cold chamber

olratio $=1.54 \times 10^{15}\left(\mathrm{ohm}-\mathrm{m}^{2}\right)^{-1}$

$s_{a}=10 \mu \mathrm{m}$

Broadband Resonator

$R_{\perp}=1.0 \mathrm{M} \Omega / \mathrm{m}$

$Q_{\perp}=1$

$\omega_{r}=2 \pi \times 10^{9} f_{r}(G h z)$

$W_{0}^{\text {res }}=\frac{\omega_{r} R_{\perp}}{\sqrt{Q_{\perp}^{2}-\frac{1}{4}}}=1.1 \times 10^{16} f_{r}(G H z) \frac{\Omega}{m-s}$

$\mathrm{Y}^{r e s}=\frac{e^{2} N_{e} \beta_{y} W_{0}^{\text {res }}}{8 \gamma m c^{2} v_{s}}=0.34 f_{r}(G H z)$

Normal Resistive Wall

$W_{0}^{r w}=\frac{c Z_{0}}{4 \pi} \frac{2 s_{0} L}{b^{4}}=3.7 \times 10^{17} \frac{\Omega}{m-s}$,

$\mathrm{Y}^{r w}=\frac{e^{2} N_{e} \beta_{y} W_{0}^{r w}}{8 \gamma m c^{2} v_{s}}=17.4$

Extreme Anomalous Skin Effect

$W_{0}^{\text {ease }}=\frac{c Z_{0}}{4 \pi} \frac{2 s_{a} L}{b^{4}}$,

$$
\mathrm{Y}^{\text {ease }}=\frac{e^{2} N_{e} \beta_{y} W_{0}^{\text {ease }}}{8 \gamma m c^{2} v_{s}}=27.3
$$




\section{Simulation Results: Broad Band Resonance, Fundamental RF}

Resonator: $R_{\perp}=1.0 \mathrm{M} \Omega / \mathrm{m}, \quad Q_{\perp}=1, \quad f_{r}=10 \mathrm{Ghz}$

$n_{p}=100, \quad n_{r}=10000$

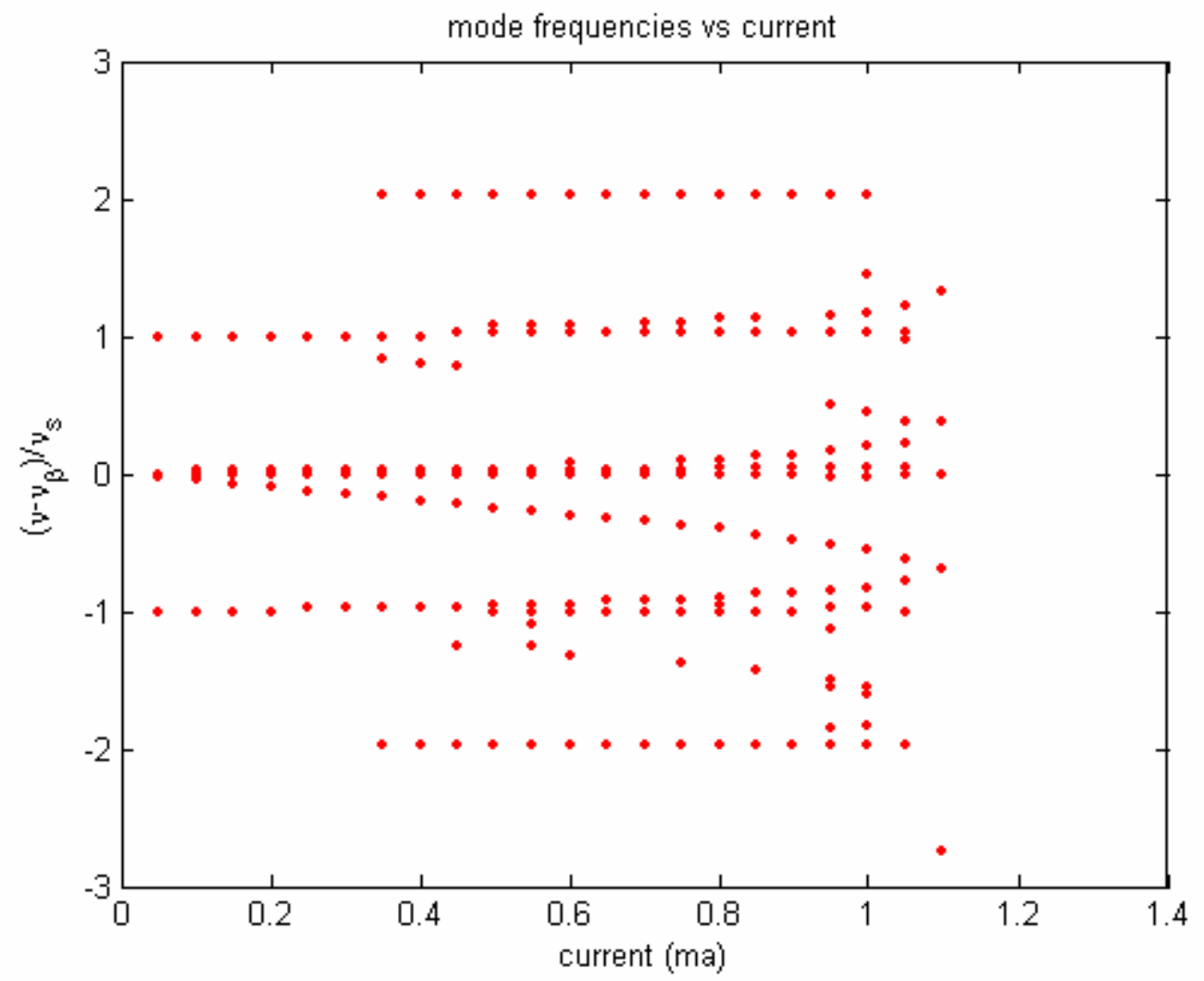

threshold current between 1.05 and $1.10 \mathrm{ma}$ 

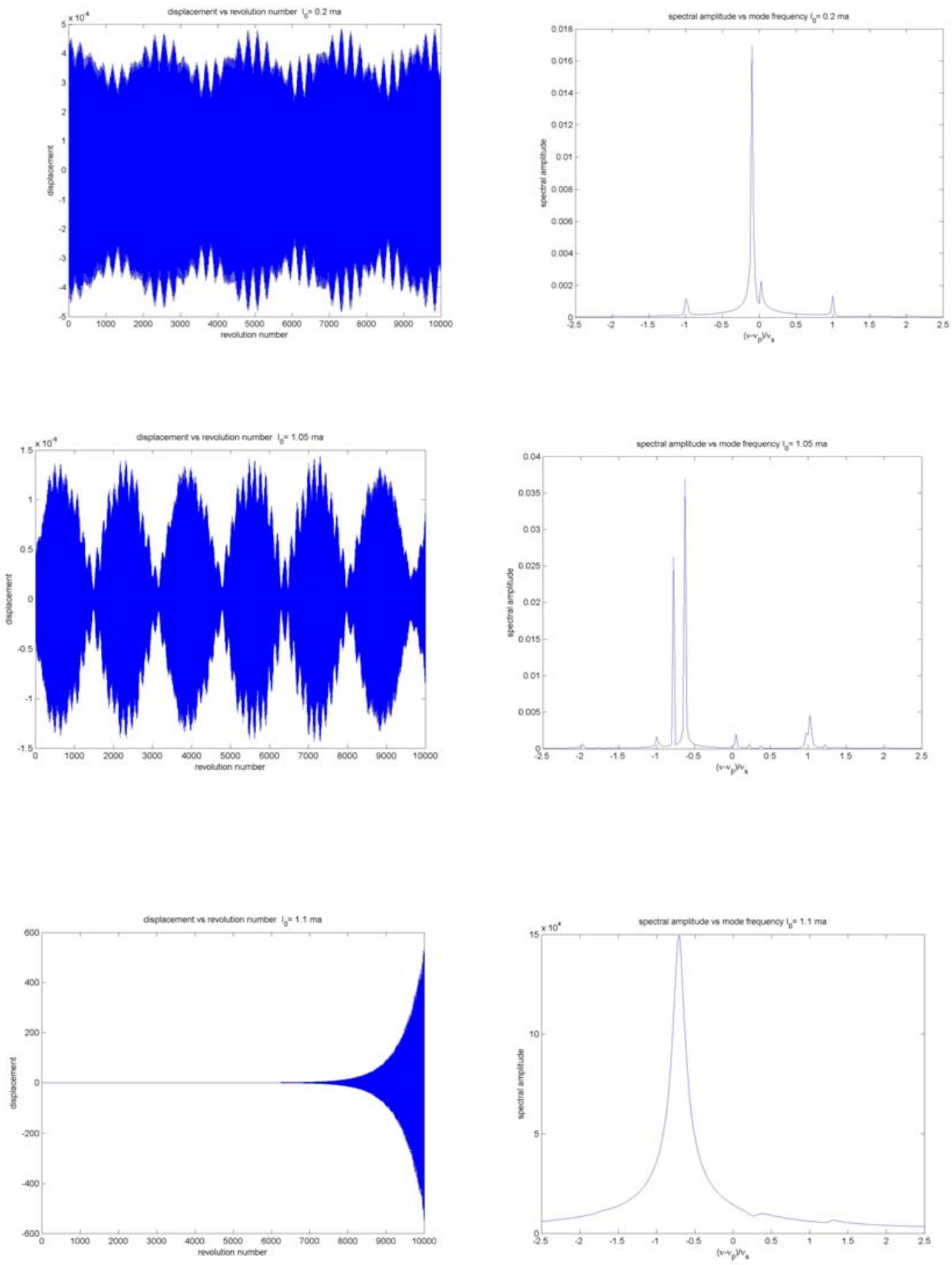
Resonator: $R_{\perp}=1.0 \mathrm{M} \Omega / \mathrm{m}, \quad Q_{\perp}=1, \quad f_{r}=30 G \mathrm{hz}$

$$
n_{p}=300, \quad n_{r}=10000
$$

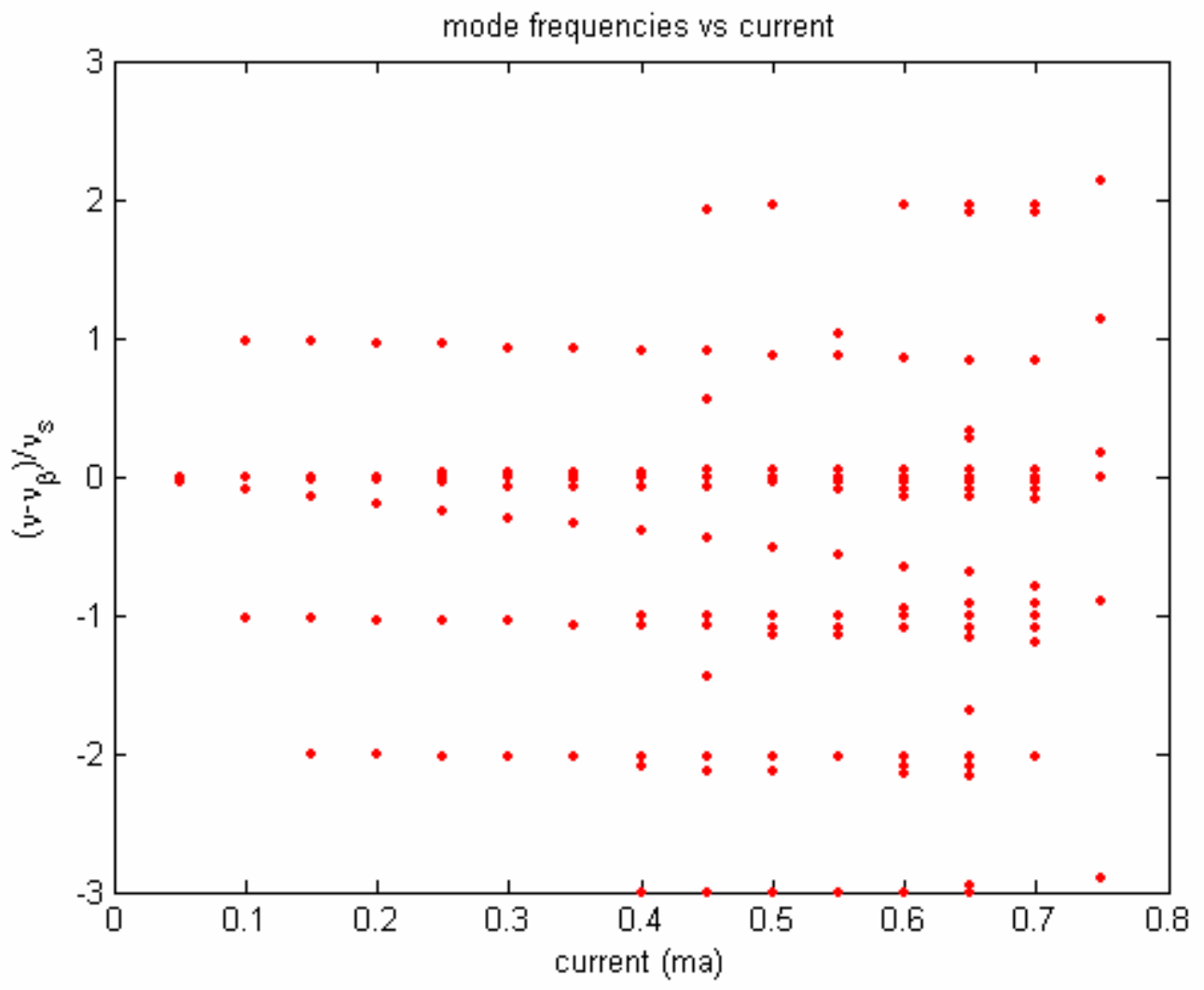

threshold current between 0.70 and $0.75 \mathrm{ma}$ 
Resonator: $R_{\perp}=1.0 \mathrm{M} \Omega / m, \quad Q_{\perp}=1, \quad f_{r}=50 G h z$

$$
n_{p}=500, \quad n_{r}=10000
$$

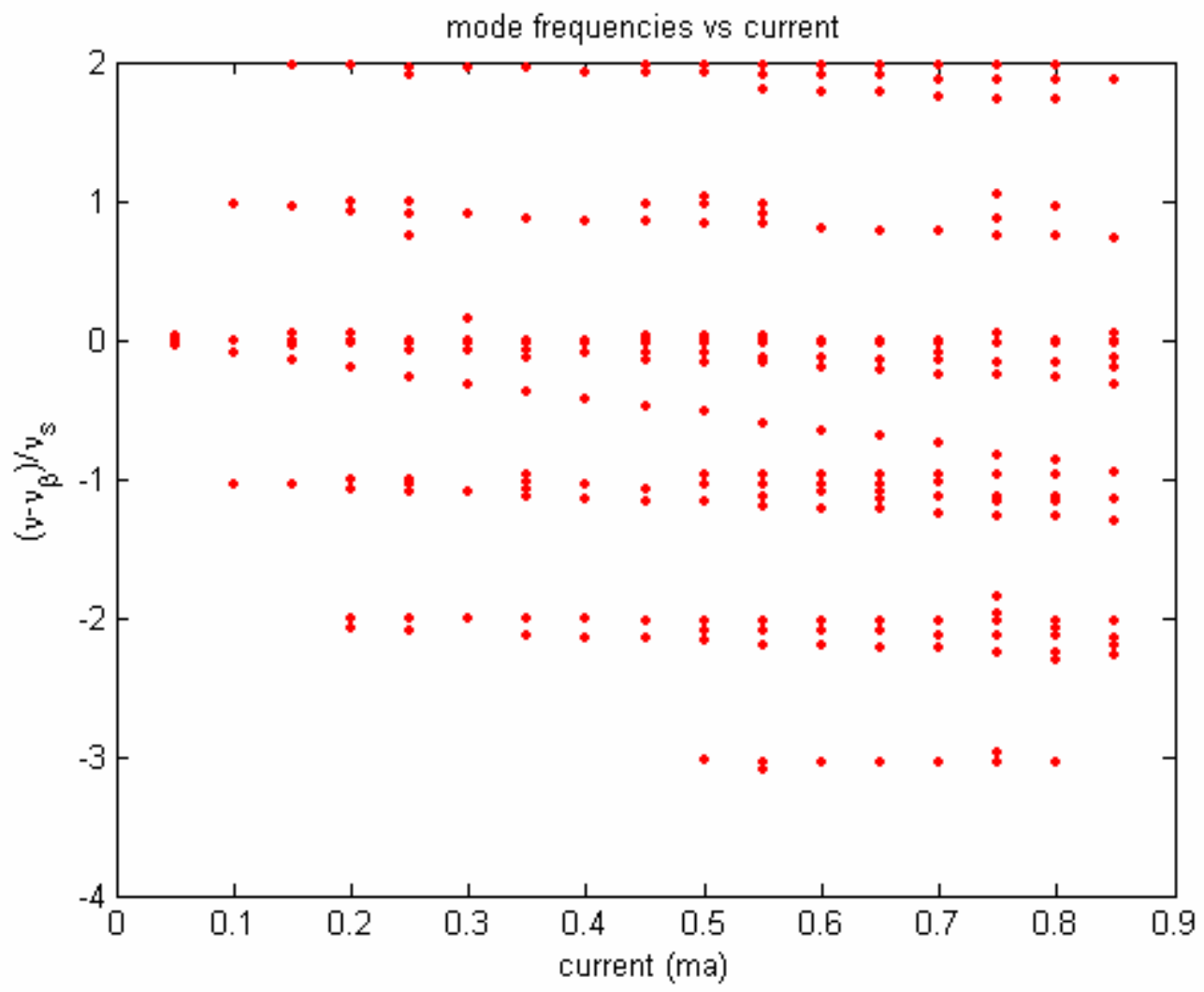

threshold current between 0.80 and 0.85 ma 
Resonator: $R_{\perp}=1.0 \mathrm{M} \Omega / m, \quad Q_{\perp}=1, \quad f_{r}=100 \mathrm{Ghz}$

$$
n_{p}=1000, \quad n_{r}=10000
$$

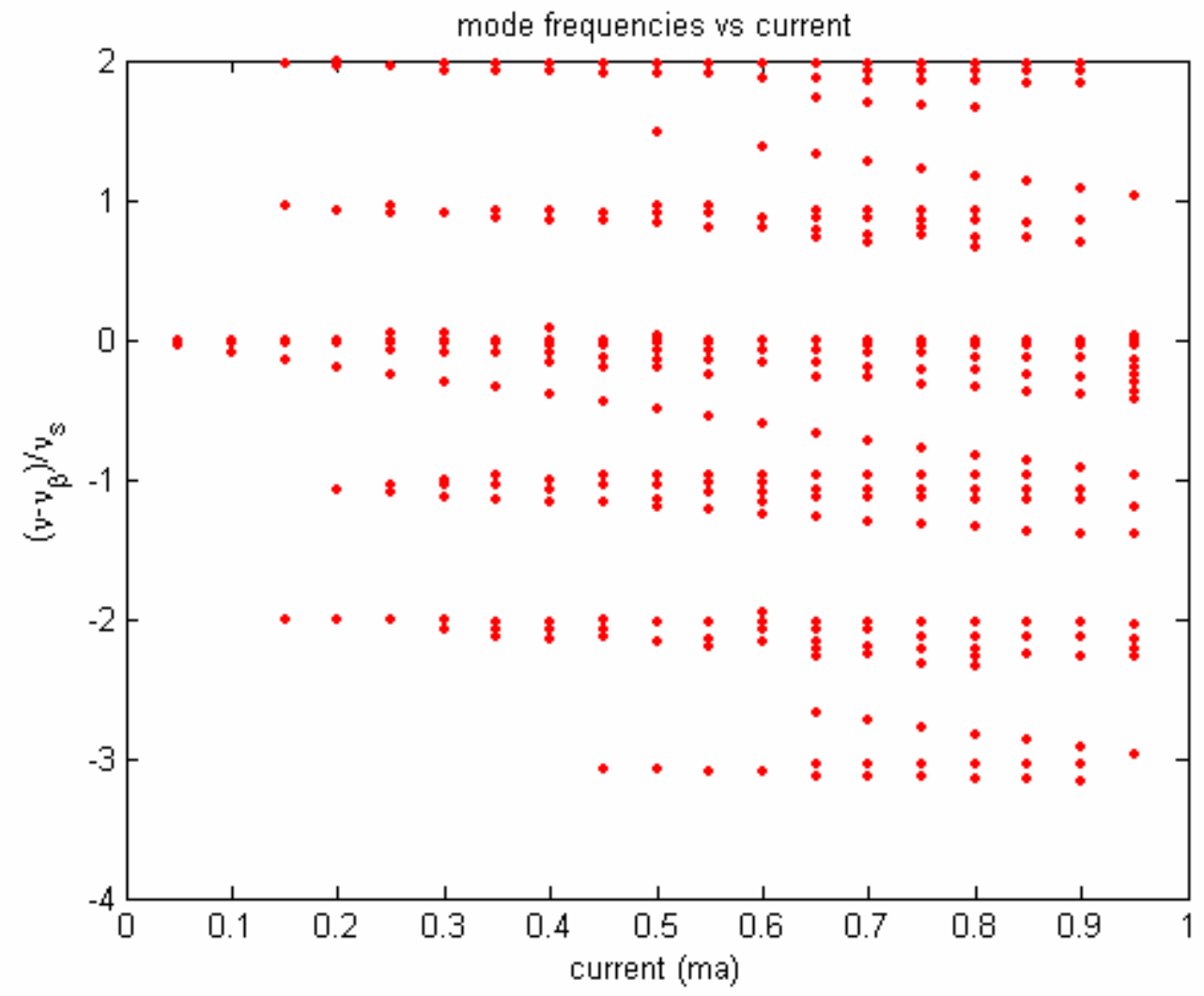

threshold current between 0.90 and $0.95 \mathrm{ma}$ 

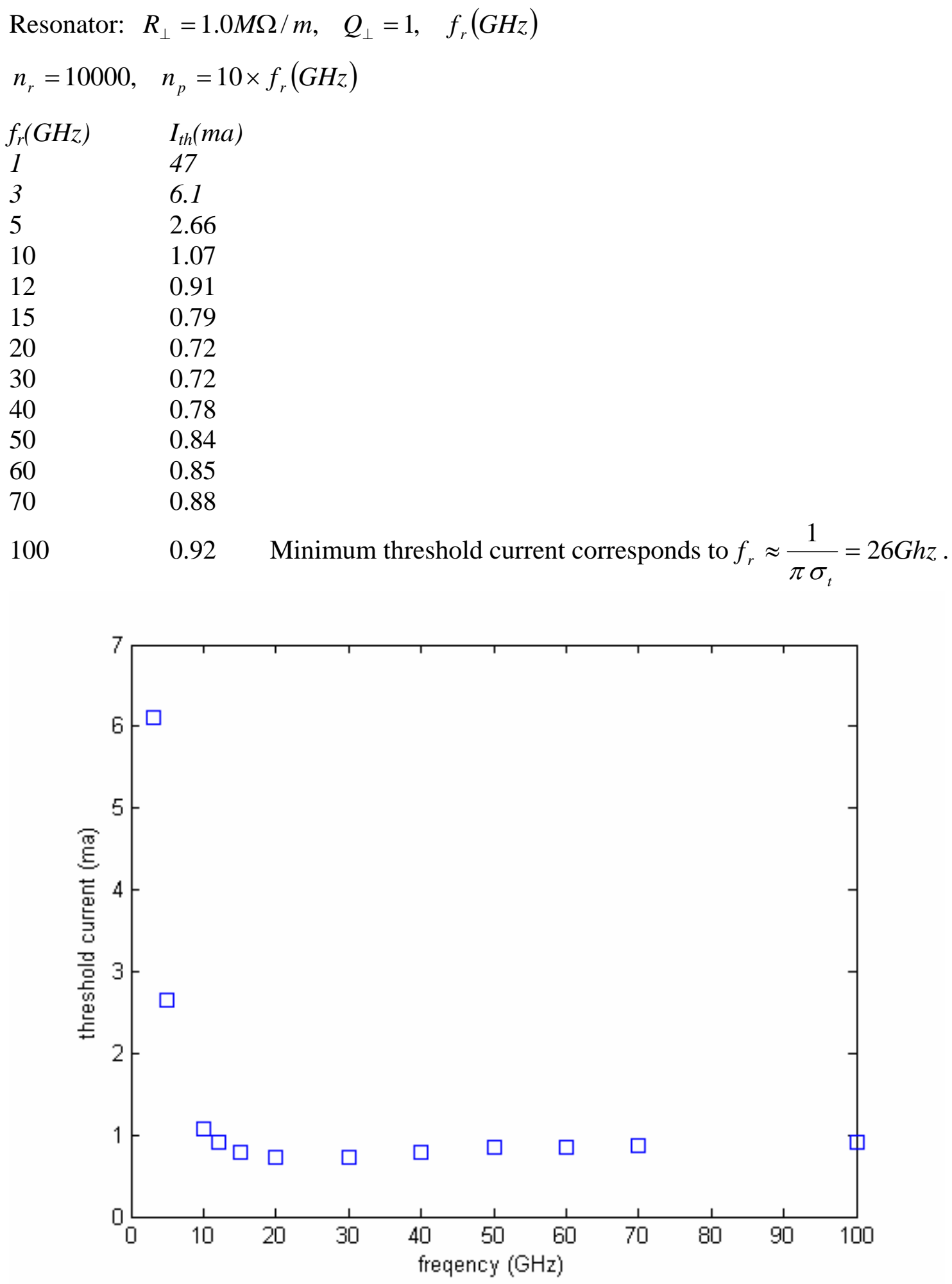

I have checked that in this case of a broadband resonator wake and the fundamental RF, my simulation results are in reasonable agreement with the frequency domain code MOSES [4]. 


$$
\begin{aligned}
& \left(\frac{\Delta v_{y}}{v_{s}}\right)_{a v}^{r e s}=\frac{2}{\pi} \mathrm{Y}_{t h} \frac{1}{2 \pi \sigma_{s}^{2}} \int_{-\infty}^{\infty} d s \int_{-\infty}^{s} d s^{\prime} e^{-s^{2} / 2 \sigma_{s}^{2}} e^{-s^{2^{2}} / 2 \sigma_{s}^{2}} f^{r e s}\left(s-s^{\prime}\right) \\
& \mathrm{Y}_{\text {th }}^{r e s}=\frac{e^{2} N_{e}^{\text {th }} \beta_{y}}{8 \gamma m c^{2} v_{s}} \frac{2 \pi f_{r} R_{\perp}}{\sqrt{Q_{\perp}^{2}-\frac{1}{4}}}=0.34 \frac{I_{t h}(m a) f_{r}(G H z)}{0.75} \\
& I_{t h}(m a)=\frac{10.2}{f_{r}(G H z) h\left[0.024 \pi f_{r}(G H z)\right]} \\
& h\left(k_{r} \sigma_{s}\right)=\int_{-\infty}^{\infty} d x \int_{-\infty}^{x} d y e^{-\left(x^{2}+y^{2}\right) / 2} \exp \left[-\frac{k_{r} \sigma_{s}}{2 Q_{\perp}}(x-y)\right] \sin \left[k_{r} \sigma_{s} \sqrt{1-\frac{1}{4 Q_{\perp}^{2}}}(x-y)\right]
\end{aligned}
$$

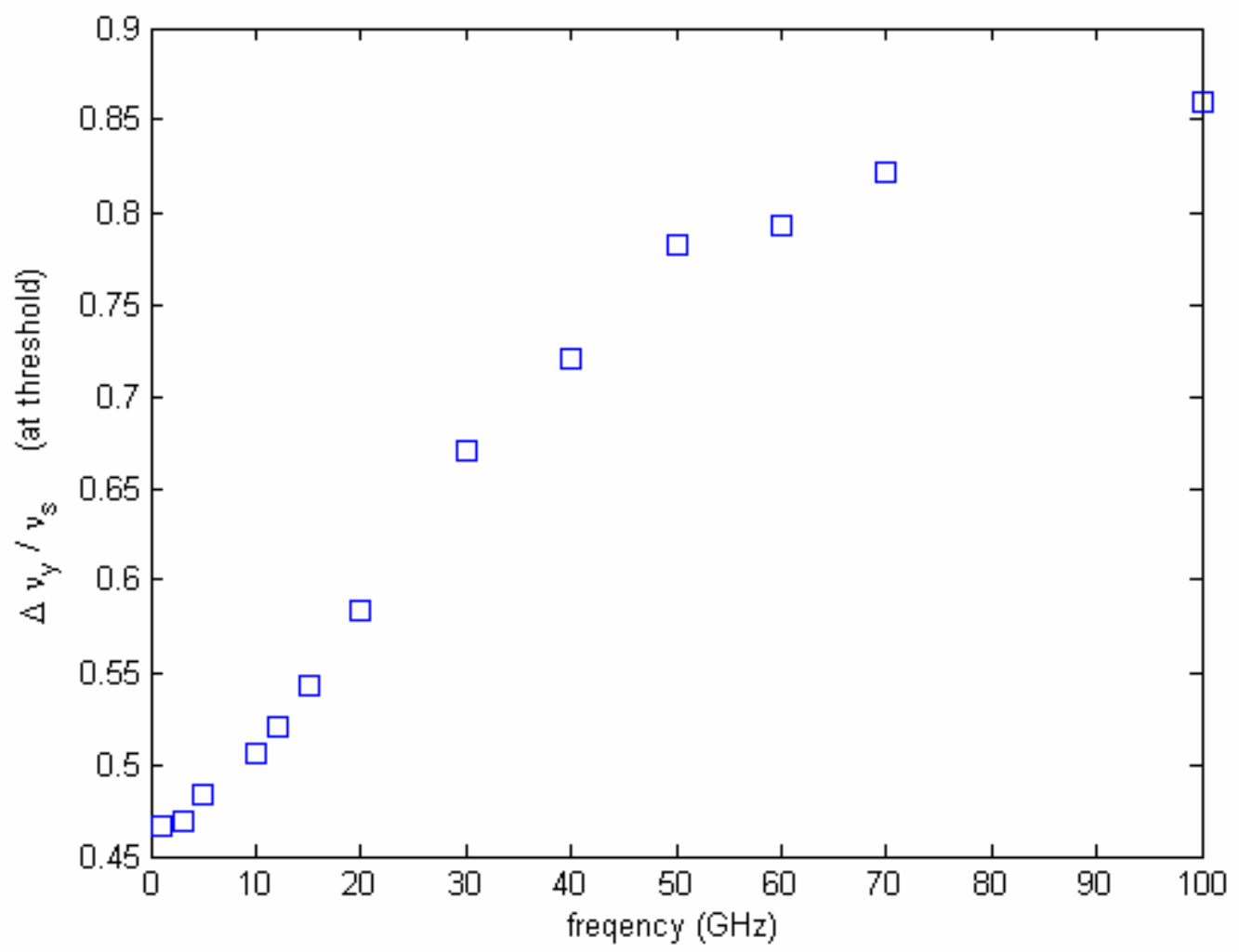




\section{Simulation Results: Resistive Wall, Fundamental RF}

Resistive Wall: $n_{r}=10000$

$\begin{array}{rl}n_{p} & I_{t h} \\ 100 & 1.66 m a \\ 200 & 1.70 \\ 400 & 1.71\end{array}$

Resistive wall: $n_{p}=200, \quad n_{r}=10000$

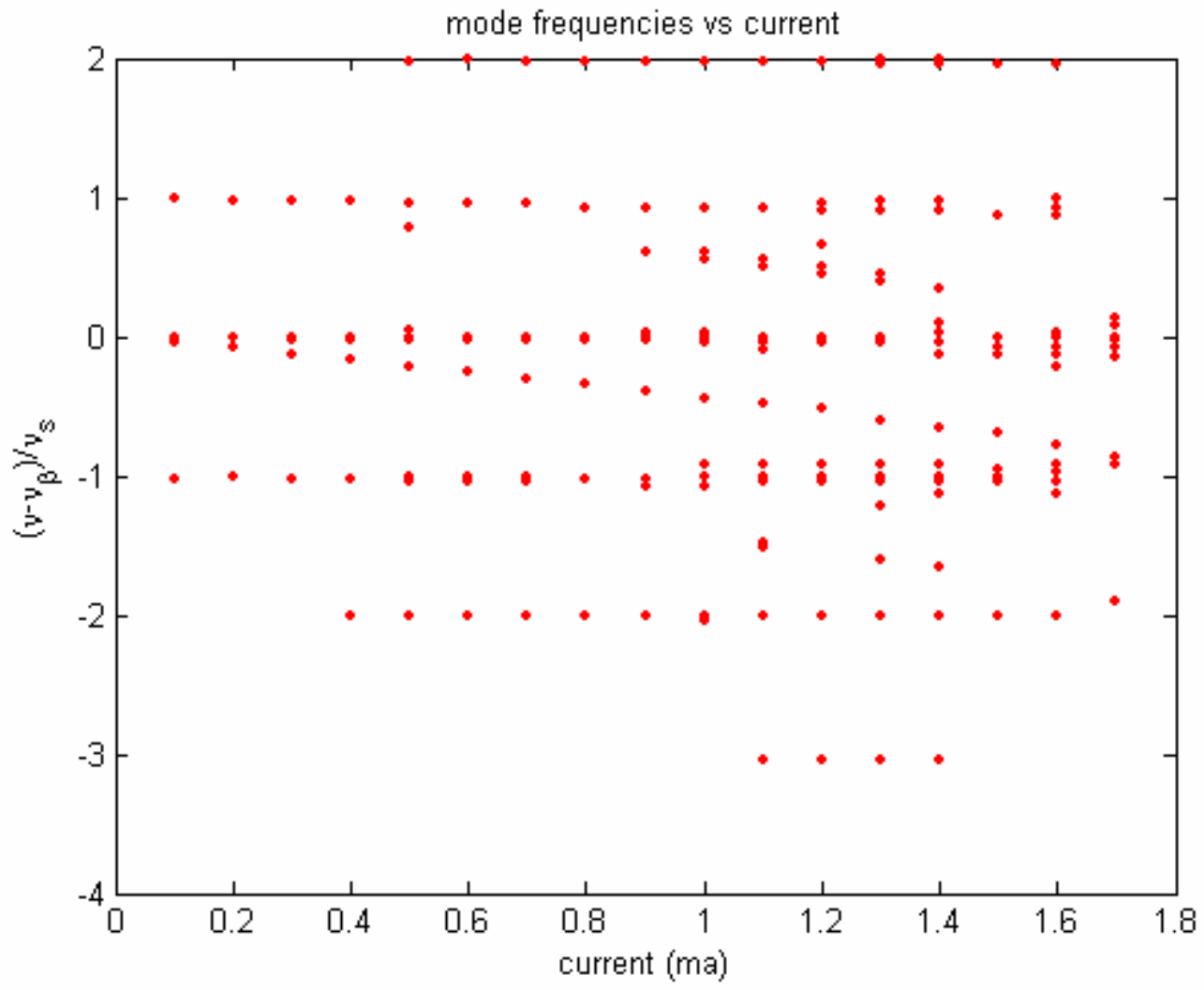

$$
\begin{aligned}
\left(\frac{\Delta v_{y}}{v_{s}}\right)_{a v} & =\frac{2}{\pi} \mathrm{Y}^{r w} \frac{1}{2 \pi \sigma_{s}^{2}} \int_{-\infty}^{\infty} d s \int_{-\infty}^{s} d s^{\prime} e^{-s^{2} / 2 \sigma_{s}^{2}} e^{-s^{\prime 2} / 2 \sigma_{s}^{2}} \sqrt{\frac{2 s_{0}}{\pi\left(s-s^{\prime}\right)}} \\
& =0.37 \mathrm{Y}^{r w} \sqrt{\frac{s_{0}}{\sigma_{s}}}
\end{aligned}
$$

Threshold, $\mathrm{Y}_{t h}^{r w}=17.4(1.7 / .75)=39.4$, corresponds to $\left(\frac{\Delta v_{y}}{v_{s}}\right)_{a v}=0.69$. 


\section{Simulation Results: Extreme Anomalous Skin Effect, Fundamental RF}

Extreme Anomalous Skin Effect: $n_{r}=10000$

$\begin{array}{cc}n_{p} & I_{\text {th }} \\ 100 & 6.36 \\ 200 & 6.74 \\ 400 & 6.79\end{array}$

Extreme Anomalous Skin Effect: $n_{p}=200, \quad n_{r}=10000$

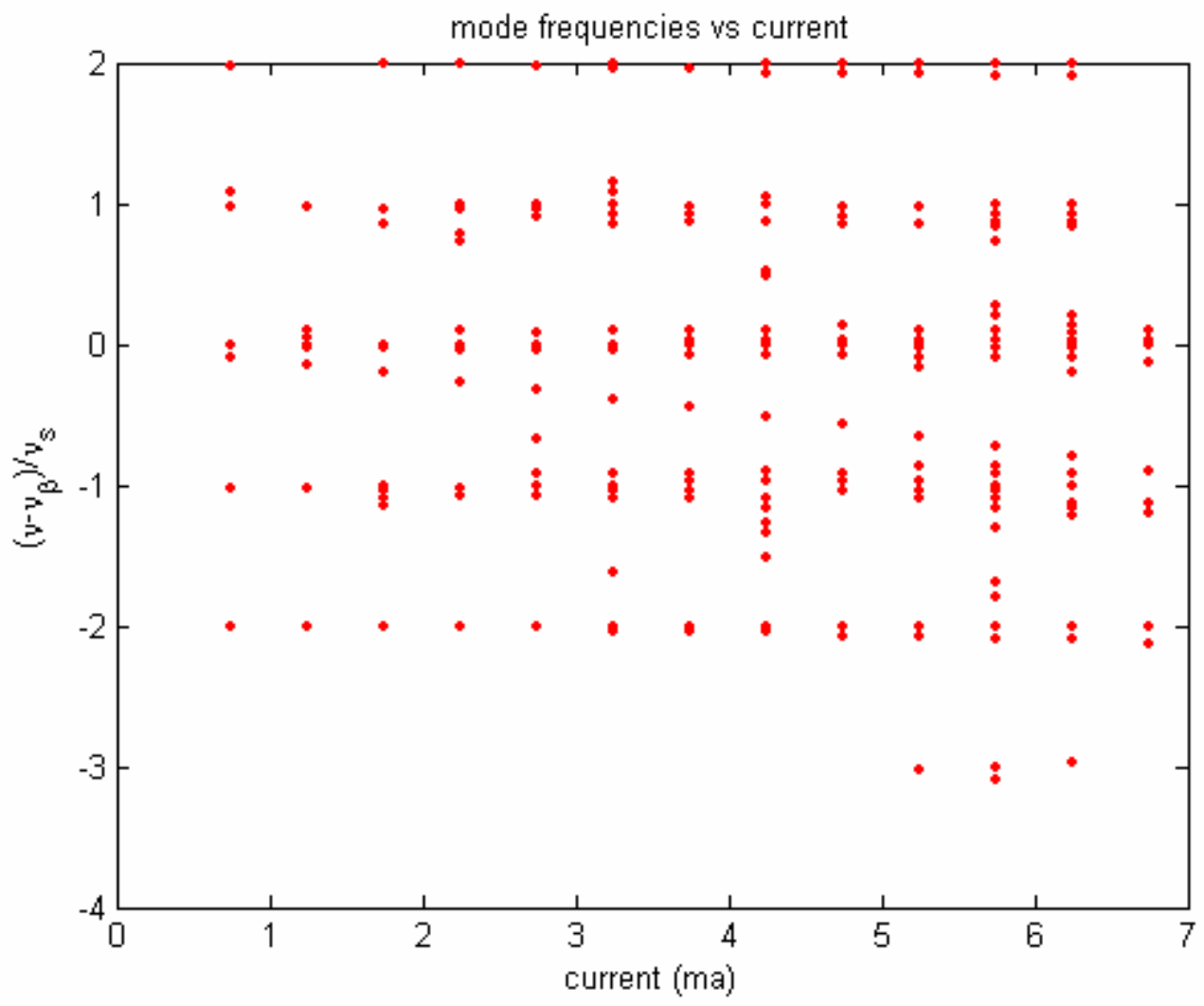

$$
\begin{aligned}
& \eta=\frac{4 \sin ^{5 / 3}(\pi / 10)}{\Gamma(1 / 3)}=0.210 \\
& \left(\frac{\Delta v_{y}}{v_{s}}\right)_{a v}=\frac{2}{\pi} \mathrm{Y}^{\text {esa }} \frac{1}{2 \pi \sigma_{s}^{2}} \int_{-\infty}^{\infty} d s \int_{-\infty}^{s} d s^{\prime} e^{-s^{2} / 2 \sigma_{s}^{2}} e^{-s^{\prime 2} / 2 \sigma_{s}^{2}} \eta\left(\frac{s_{a}}{s-s^{\prime}}\right)^{2 / 3}=0.13 \mathrm{Y}^{\text {ease }}\left(\frac{s_{a}}{\sigma_{s}}\right)^{2 / 3}
\end{aligned}
$$

Threshold, $\mathrm{Y}_{t h}^{\text {ease }}=27.3(7.79 / .75)=247$, corresponds to $\left(\frac{\Delta v_{y}}{v_{s}}\right)_{a v}=0.64$. 


\section{Third-Harmonic (Landau) Cavity}

Let us begin by reviewing the operation of a harmonic, bunch-lengthening cavity. The fundamental cavity operates at an angular frequency, $\omega_{r f}=h \omega_{0}$, where $h$ is an integer and $\omega_{0}=2 \pi / T_{0}$ is the angular frequency of revolution. Assuming the Landau cavity is operating at the $n^{\text {th }}$ harmonic of the frequency of the fundamental RF, the voltage seen by an electron with temporal deviation $\tau$ is

$$
V(\tau)=V_{0}\left[\sin \left(\omega_{r f} \tau+\phi_{s}\right)+\kappa \sin \left(n \omega_{r f}+\phi_{n}\right)\right] .
$$

In the ideal operation of such a cavity, one chooses to satisfy the conditions

$$
\begin{aligned}
& U_{0}=V_{0}\left[\sin \phi_{s}+\kappa \sin \phi_{n}\right] \\
& 0=\cos \phi_{s}+n \kappa \cos \phi_{n} \\
& 0=\sin \phi_{s}+n \kappa \sin \phi_{n} .
\end{aligned}
$$

In this case, the voltage has the form

$$
V(\tau)=\cos \phi_{s}\left(\sin \omega_{r f} \tau-\frac{1}{n} \sin n \omega_{r f} \tau\right)+\sin \phi_{s}\left(\cos \omega_{r f} \tau-\frac{1}{n^{2}} \cos n \omega_{r f} \tau\right) .
$$

Approximating this for small $\tau$ yields the cubic form

$$
V(\tau) \cong \cos \phi_{s}\left(\frac{n^{2}-1}{6}\right)\left(\omega_{r f} \tau\right)^{3}+\sin \phi_{s}\left(1-\frac{1}{n^{2}}\right) .
$$

The equations of motion become

$$
\begin{aligned}
& \dot{\tau}=-\alpha \varepsilon \\
& \dot{\varepsilon}=\frac{e V_{0} \cos \phi_{s}}{E_{0} T_{0}}\left(\frac{n^{2}-1}{6}\right)\left(h \omega_{0} \tau\right)^{3} .
\end{aligned}
$$

Together, these two equations imply

$$
\ddot{\tau}+\rho \tau=0 .
$$

where

$$
\rho=v_{r e f}^{2} h^{2}\left(\frac{n^{2}-1}{6}\right) \omega_{0}^{4}
$$

and

$$
v_{r e f}^{2} \equiv \frac{h \alpha e V_{0} \cos \phi_{s}}{2 \pi E_{0}}
$$


The solution to Eq. (8.6) can be written in terms of an elliptic function of modulus 1/2,

$$
\tau(t)=\operatorname{rcn}\left(\sqrt{\rho} r t ; k^{2}=1 / 2\right) \text {. }
$$

The synchrotron frequency has a linear dependence on the oscillation amplitude $r$,

$$
\omega_{s}=\frac{\pi}{2 K} \sqrt{\rho} r
$$

where the elliptic integral $K=K\left(k^{2}=1 / 2\right)=\frac{\sqrt{2 \pi} \Gamma(1 / 4)}{4 \Gamma(3 / 4)}=1.85$.

The equilibrium distribution determined by radiation damping and quantum fluctuations is proportional to $\exp \left[-\frac{1}{\sigma_{\varepsilon}^{2}}\left(\frac{\varepsilon^{2}}{2}+\frac{\rho \tau^{4}}{4 \alpha^{2}}\right)\right]$, and the rms bunch duration $\Sigma_{t}$ is given by

$$
\Sigma_{t}^{2}=\frac{\Gamma(3 / 4)}{\Gamma(1 / 4)} \frac{2 \alpha \sigma_{\varepsilon}}{\sqrt{\rho}}
$$

It is useful to define $\bar{v}_{s}$ to be the synchrotron tune corresponding to a synchrotron oscillation amplitude equal to the rms bunch duration, $\Sigma_{t}$, i.e.

$$
\bar{v}_{s} \equiv \frac{\pi}{2 K} \sqrt{\frac{\rho}{\omega_{0}^{2}}} \Sigma_{t} .
$$

Then one can write

$$
\bar{v}_{s} \omega_{0} \Sigma_{t}=\left(\frac{\pi}{2}\right)^{3 / 2} \frac{\alpha}{K^{2}} \sigma_{\varepsilon}
$$

For NSLS-II parameters:

\begin{tabular}{|l|l|}
\hline $\mathrm{h}$ & 1000 \\
\hline $\mathrm{n}$ & 3 \\
\hline$v_{r e f}$ & 0.004 \\
\hline$\rho$ & $2.1 \times 10^{27} \mathrm{sec}^{-4}$ \\
\hline$\Sigma_{t} / \Sigma_{s}$ & $50 \mathrm{ps} / 15 \mathrm{~mm}$ \\
\hline $\bar{v}_{s}$ & 0.0006 \\
\hline
\end{tabular}


In order to carry out simulations to study the transverse instabilities with a thirdharmonic Landau cavity, we use Eqs.(2.5-2.10) and replace Eq. (2.4) by

$$
\varepsilon_{i+1}(j, k)=\varepsilon_{i}(j, k)+\frac{b}{\alpha T_{0}^{3}} \tau_{i}(j, k)^{3},
$$

where

$$
b=\rho T_{0}^{4}=\left[\frac{2 \Gamma(3 / 4) \alpha \sigma_{\varepsilon} T_{0}^{2}}{\Gamma(1 / 4) \Sigma_{t}^{2}}\right]^{2} .
$$

The initial conditions for the transverse motion are determined in the same manner as for the case when there is only the fundamental RF cavity. However, in order to determine initial conditions for the longitudinal motion, we carry out a short simulation to obtain a thermal distribution. In order to accomplish this, we use the equations of motion:

$$
\begin{aligned}
& \varepsilon_{n+1}=\varepsilon_{n}+\frac{b \tau_{n}^{3}}{\alpha T_{0}^{3}}-a \varepsilon_{n}-u_{n} \\
& \tau_{n+1}=\tau_{n}-\alpha T_{0} \varepsilon_{n+1}
\end{aligned}
$$

Here, $a$ is the inverse damping time and $u_{n}$ is Gaussian random variable with standard deviation

$$
\sigma_{u}=\sqrt{2 a} \sigma_{\varepsilon}
$$

We start this simulation with $\varepsilon_{n}, \tau_{n}$ distributed according to a Gaussian distribution. The final equilibrium values are very insensitive to the starting values. We then use the final values as initial conditions for the simulation of the transverse instabilities with a Landau cavity. 


\section{Simulation Results: Broad Band Resonance, Landau Cavity}

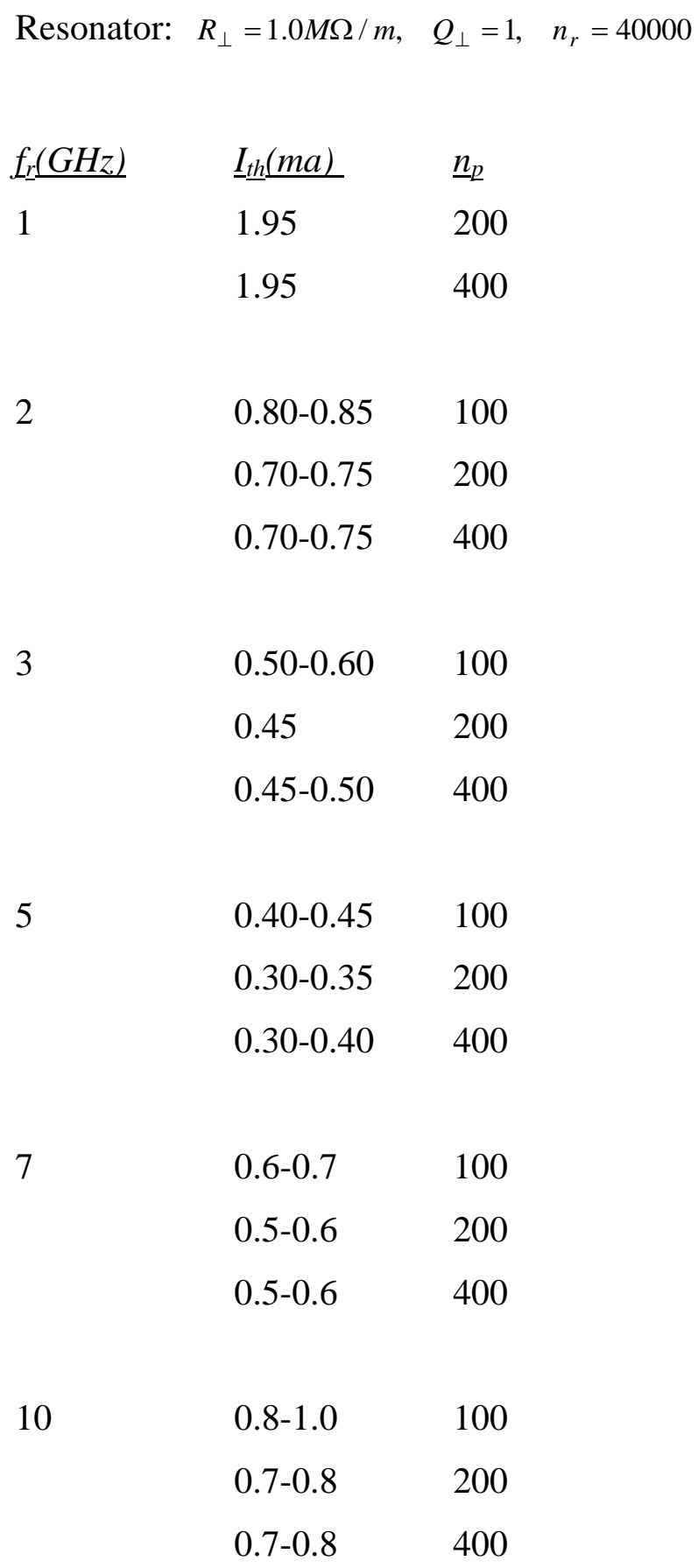

complicated behavior observed, slow growth rate near threshold, sometimes stable region found at higher current than first observation of instability, 


$$
\begin{aligned}
& \hat{N}=\frac{2 \lambda^{1 / 4}}{\Gamma(1 / 4)} \quad \Sigma_{s}^{2}=\frac{\Gamma(3 / 4)}{\lambda^{1 / 2} \Gamma(1 / 4)} \quad \lambda=\frac{\rho}{4 \alpha^{2} \sigma_{\varepsilon}^{2} c^{4}} \\
& \left(\frac{\Delta v_{y}}{v_{s}}\right)_{a v}=\left(\frac{2}{\pi}\right) \mathrm{Y}_{r e f}^{r w} \frac{v_{s}^{r e f}}{v_{s}} \hat{N}^{2} \int_{-\infty}^{\infty} d s \int_{-\infty}^{s} d s^{\prime} e^{-\lambda\left(s^{4}+s^{\prime 4}\right)} f^{r e s}\left(s-s^{\prime}\right) \\
& =\left(\frac{2}{\pi}\right) \mathrm{Y}_{r e f}^{r w} \frac{v_{s}^{r e f}}{v_{s}} \frac{4}{\Gamma^{2}(1 / 4)} h\left(k \lambda^{-1 / 4}\right) \\
& h(\kappa)=\int_{-\infty}^{\infty} d x \int_{-\infty}^{x} d y e^{-\left(x^{4}+y^{4}\right)} \exp \left[-\frac{\kappa}{2 Q_{\perp}}(x-y)\right] \sin \left[\kappa \sqrt{1-\frac{1}{4 Q_{\perp}^{2}}}(x-y)\right]
\end{aligned}
$$

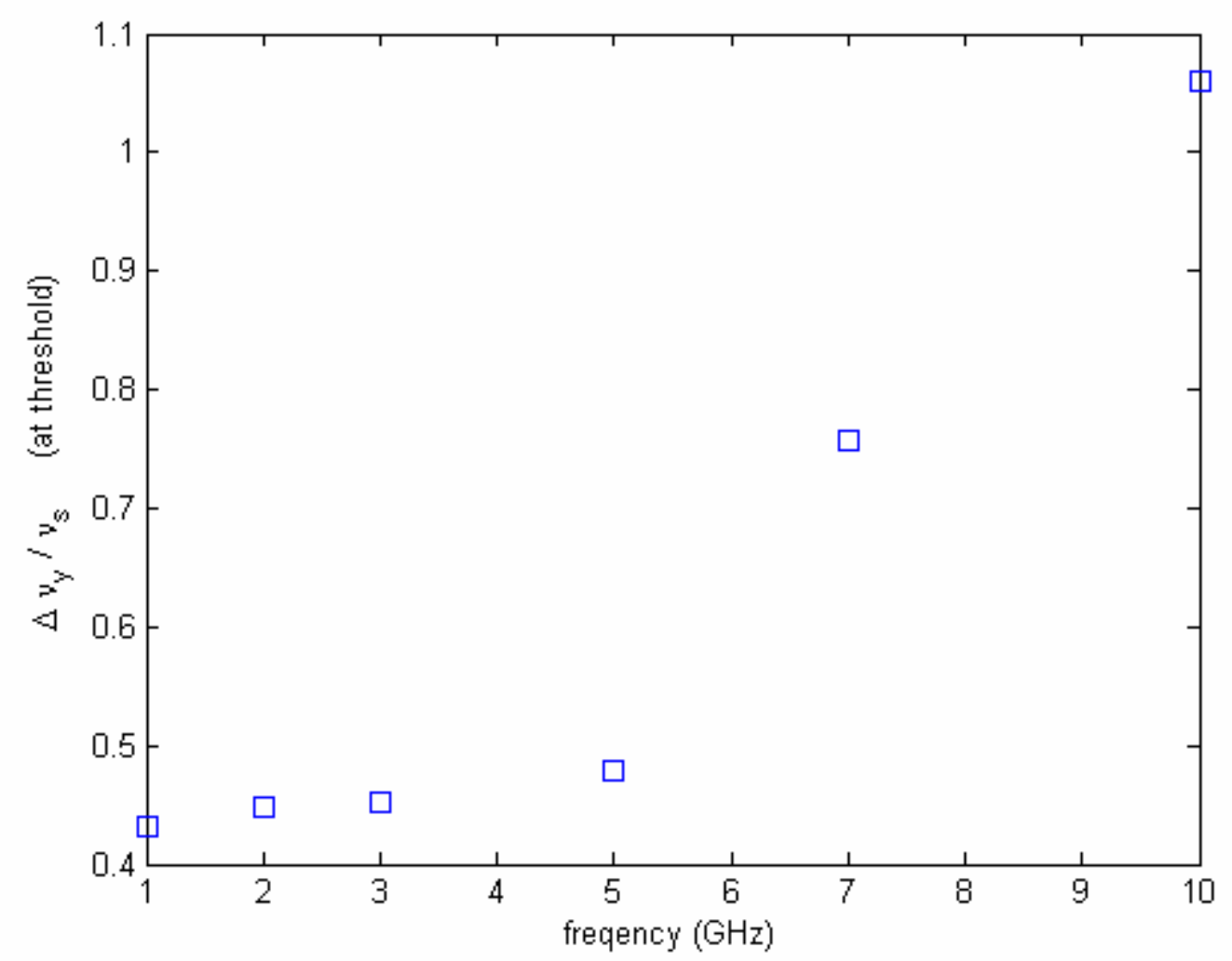




\section{Simulation Results: Resistive Wall, Landau Cavity}

Resistive Wall: $n_{r}=40000$

$\begin{array}{rl}n_{p} & I_{t h} \\ 100 & 0.65 \mathrm{ma} \\ 200 & 0.46 \\ 400 & 0.46\end{array}$

$\hat{N}=\frac{2 \lambda^{1 / 4}}{\Gamma(1 / 4)} \quad \Sigma_{s}^{2}=\frac{\Gamma(3 / 4)}{\lambda^{1 / 2} \Gamma(1 / 4)} \quad \lambda=\frac{\rho}{4 \alpha^{2} \sigma_{\varepsilon}^{2} c^{4}}$

$\left(\frac{\Delta v_{y}}{\bar{v}_{s}}\right)_{a v}=\left(\frac{2}{\pi}\right) \mathrm{Y}_{r e f}^{r w} \frac{v_{s}^{r e f}}{\bar{v}_{s}} \hat{N} \int_{-\infty}^{2} d s \int_{-\infty}^{s} d s^{\prime} e^{-\lambda\left(s^{4}+s^{\prime 4}\right)} \sqrt{\frac{2 s_{0}}{\pi\left(s-s^{\prime}\right)}}$

$=\left(\frac{2}{\pi}\right) \mathrm{Y}_{r e f}^{r w} \frac{v_{s}^{r e f}}{\nu_{s}}\left(\hat{N} \lambda^{-1 / 4}\right)^{2} \sqrt{\frac{2 s_{0}}{\pi \lambda^{-1 / 4}}}(3.05)$

$=\left(\frac{2}{\pi}\right) \mathrm{Y}_{r e f}^{r w} \frac{v_{s}^{r e f}}{\nu_{s}} \frac{4}{\Gamma^{2}(1 / 4)}\left[\frac{\Gamma(3 / 4)}{\Gamma(1 / 4)}\right]^{1 / 4} \sqrt{\frac{2 s_{0}}{\pi \Sigma_{s}}}(3.05)$

$=0.36 \mathrm{Y}_{r e f}^{r w} \frac{v_{s}^{r e f}}{v_{s}} \sqrt{\frac{s_{0}}{\Sigma_{s}}}$

Threshold, $\mathrm{Y}_{r e f, t h}^{r w}=17.4 \frac{0.46}{0.75}=10.7$, corresponds to $\left(\frac{\Delta v_{y}}{\overline{v_{s}}}\right)_{a v}=0.59$. 


\section{Simulation Results: Extreme Anomalous Skin Effect, Landau Cavity}

Extreme Anomalous Skin Effect: $n_{r}=40000$

$n_{p} \quad I_{t h}$

$200 \quad 2.3$

$400 \quad 2.4$

$$
\begin{aligned}
& \hat{N}=\frac{2 \lambda^{1 / 4}}{\Gamma(1 / 4)} \quad \Sigma_{s}^{2}=\frac{\Gamma(3 / 4)}{\lambda^{1 / 2} \Gamma(1 / 4)} \quad \lambda=\frac{\rho}{4 \alpha^{2} \sigma_{\varepsilon}^{2} c^{4}} \\
& \left(\frac{\Delta v_{y}}{\bar{v}_{s}}\right)_{a v}=\left(\frac{2}{\pi}\right) \mathrm{Y}_{\text {ref }}^{\text {ease }} \frac{v_{s}^{r e f}}{\bar{v}_{s}} \hat{N}^{2} \int_{-\infty}^{\infty} d s \int_{-\infty}^{s} d s^{\prime} e^{-\lambda\left(s^{4}+s^{\prime 4}\right)} \eta\left(\frac{s_{a}}{s-s^{\prime}}\right)^{2 / 3} \\
& =\left(\frac{2}{\pi}\right) \mathrm{Y}_{\text {ref }}^{\text {ease }} \frac{\nu_{s}^{\text {ref }}}{\bar{\nu}_{s}}\left(\hat{N} \lambda^{-1 / 4}\right)^{2}\left(\frac{s_{a}}{\lambda^{-1 / 4}}\right)^{2 / 3} \eta(4.52) \\
& =\left(\frac{2}{\pi}\right) \mathrm{Y}_{\text {ref }}^{\text {ease }} \frac{v_{s}^{\text {ref }}}{v_{s}} \frac{4}{\Gamma^{2}(1 / 4)}\left[\frac{\Gamma(3 / 4)}{\Gamma(1 / 4)}\right]^{1 / 3}\left(\frac{s_{a}}{\lambda^{-1 / 4}}\right)^{2 / 3} \eta(4.52) \\
& =0.13 \mathrm{Y}_{\text {ref }}^{\text {ease }} \frac{v_{s}^{r e f}}{\overline{\nu_{s}}}\left(\frac{s_{a}}{\Sigma_{s}}\right)^{2 / 3}
\end{aligned}
$$

Threshold, $Y_{\text {ref } \text {,th }}^{\text {ease }}=27.3 \frac{2.4}{0.75}=87$, corresponds to $\left(\frac{\Delta v_{y}}{\bar{v}_{s}}\right)_{a v}=0.58$. 


\section{Summary of Results}

We summarize the results obtained using the NSLS-II parameters as given in Section 4. Threshold currents for different parameters can be estimated using the scaling relations (3.4) and (3.12).

\section{Fundamental RF}

Synchrotron Tune $v_{s}=.004$

Bunch length $\quad \sigma_{s}=3.6 \mathrm{~mm}$

Vertical betafunction $\beta_{y}=3 m$

Resistive wall (100m of room temperature copper, $b=2.5 \mathrm{~mm}) \quad I_{t h}(\mathrm{ma})=1.7 \mathrm{ma}$ Extreme Anomalous Skin Effect $I_{t h}(m a)=6.8 m a$

Broad-Band Resonator $R_{\perp}=1 M \Omega / m, \quad Q_{\perp}=1$

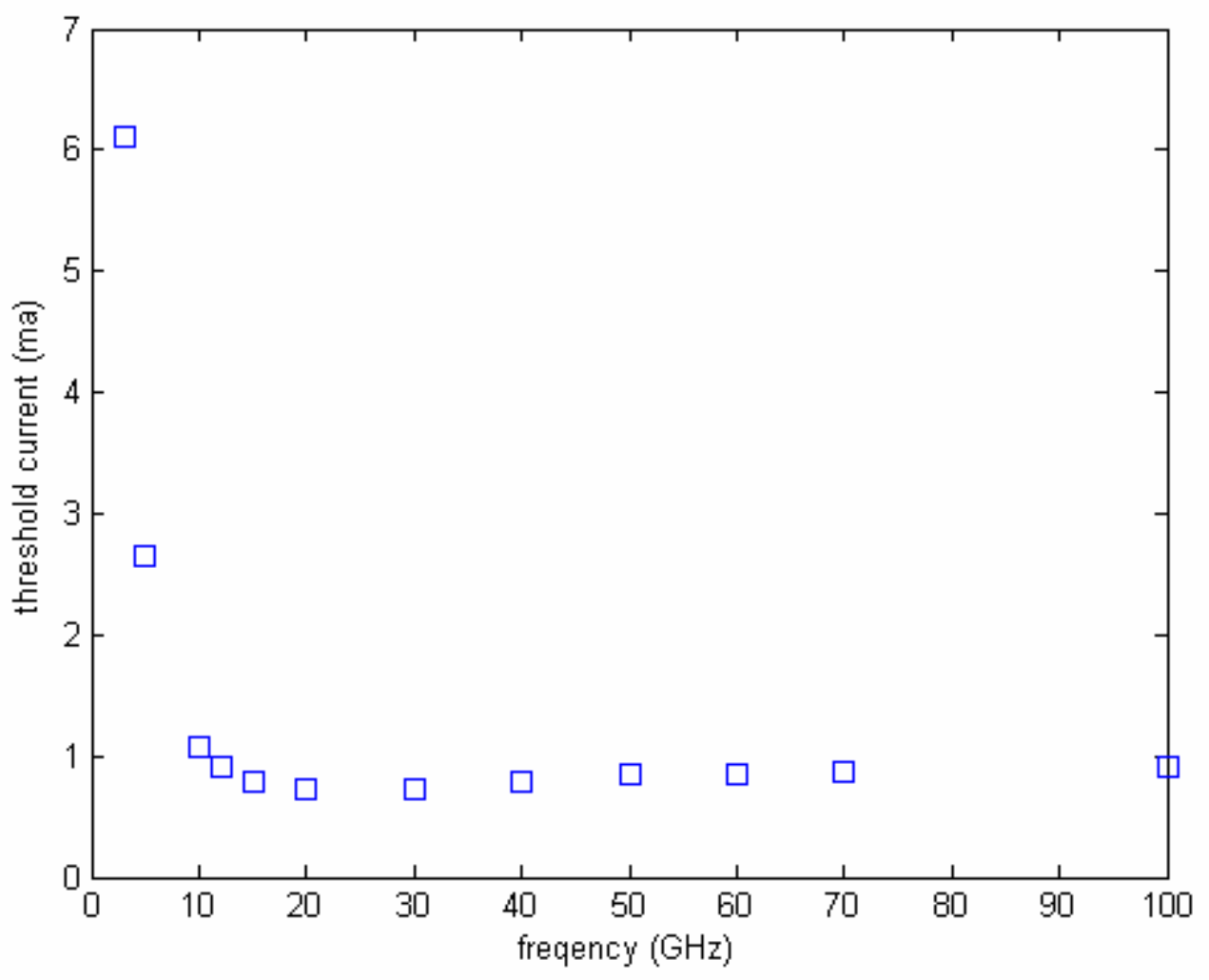




\section{Landau Cavity}

Average Synchrotron Tune $\bar{v}_{s}=.0006$

Bunch length $\quad \Sigma_{s}=15 \mathrm{~mm}$

Vertical betafunction $\quad \beta_{y}=3 m$

Resistive wall (100m of room temperature copper, $b=2.5 \mathrm{~mm}) \quad I_{t h}(\mathrm{ma})=0.46 \mathrm{ma}$

Extreme Anomalous Skin Effect $I_{t h}(m a)=2.4 m a$

Broad-Band Resonator $R_{\perp}=1 M \Omega / m, \quad Q_{\perp}=1$

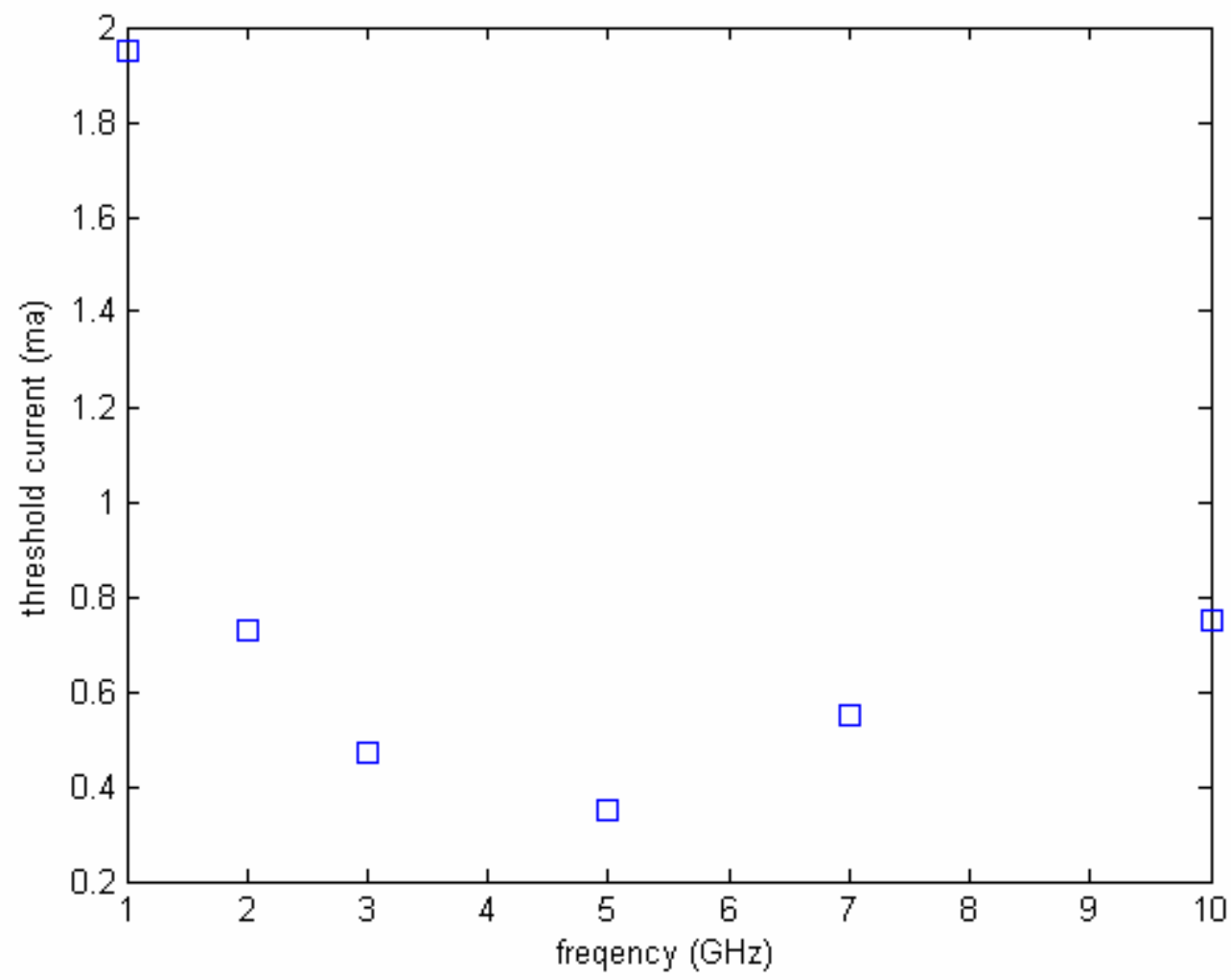




\section{Concluding Remarks}

In this note, we have described a computer simulation program written with MATLAB. Using this program, we have carried out studies of the TMCI single bunch instability for NSLS-II, in the case of vanishing chromaticity. In the future, we plan to investigate the stabilizing effect of positive chromaticity. We also plan to include the effect of wakefields coupling the center of mass motion of different bunches.

\section{Acknowledgements}

I wish to thank Boris Podobedov, Nathan Towne and Juinn Ming Wang for helpful

discussions. This work was supported by Department of Energy contract DE-AC0298CH10886. 


\section{References}

[1] A.W. Chao, Physics of Collective Beam Instabilities in High Energy Accelerators (Wiley, NY, 1993).

[2] R.D. Ruth and J.M. Wang, "Vertical Fast Blow-up in a Single Bunch,” IEEE Trans. Nucl. Sci. NS-28, 2405 (1981).

[3] Y.H. Chin, "Transverse Mode Coupling Instabilities in the SPS,” CERN/SPS/85-2.

[4] Y.H. Chin, “Users Guide for New Moses,” CERN/LEP-TH/88-05.

[5] K.C. Harkay, M. Borland, Y.C. Chae, L. Emery, Z. Huang, E.S. Lesser, A.H. Lumpkin, S.V. Milton, N.S. Sereno, B.X. Yang, "Impedance and the Single Bundh Limit in the APS Storage Ring,” Proc. PAC1999, 1644 (1999).

[6] J.L. Revol, R. Nagaoka, P. Kernel, L. Tosi, E. Karantzoulis, “Comparison of Transverse Single Bunch Instabilities Between the ESRF and ELETTRA,” Proc. EPAC2000, 1170 (2000).

[7] K. Harkay, R. Nagaoka, J.L. Revol, T. Nakamura, “A Preliminary Comparison Of Beam Instabilities Among ESRF, APS, and SPRING-8 X-Ray Storage Ring Light Sources,” Proc. EPAC2002, 1505 (2002).

[8] R. Nagaoka, “Study of Resistive-Wall Effects on SOLEIL,” Proc. EPAC 2004, 2035 (2004).

[9] G.V. Stupakov, “Wake and Impedance,” SLAC-PUB-8683 (2000).

[10] B.W. Zotter and S.A. Kheifets, Impedances and Wakes in High-Energy Particle Accelerators (World Scientific, Singapore, 1998).

[11] Y.H. Chin, "Transverse Mode Coupling Instability in a Double RF System,” Part. Accel. 45, 209 (1994).

[12] K. Bane and M. Sands, “The Short-Range Resistive Wall Wakefields,” Proc. Micro Bunches Workshop, 131 (1995).

[13] B. Podobedov, private communication. 